\title{
Interdependence of ISE 30 and Gold Spot, Natural Gas, Brent Oil: Copula-Garch Method
}

\author{
Ayşe Metin Karakaş \\ Bitlis Eren University, Faculty of Science and Art Department of Statistics, \\ Rahva Yerleskesi Bes Minare Mahallesi Ahmet Eren Bulvar 13000 Bitlis/Turkey. \\ Email: aysekarakas5767 [AT] gmail.com
}

\begin{abstract}
This paper aims to examine the relationship between ISE 30 and gold spot, natural gas and Brent Oil with the COPULA-GARCH method. In the study, we use closing prices of ISE 30 and Gold Spot, Brent Oil and Natural Gas. The results show that there is a weak dependence between ISE 30 and Brent Oil, Gold Spot but there is more strong between ISE 30 and Natural Gas.
\end{abstract}

Keywords---- ISE 30, COPULA-GARCH, Gold Spot, Brent Oil, Natural Gas

\section{INTRODUCTION}

Today, oil, an important energy source, directly or indirectly affects the economies of all countries in the world. Changes in oil prices affect the economy in various ways [1]. First, the rise in oil prices increases the cost of production which results decrease in productivity. Second, it disrupts the foreign trade balance of oil importing countries where oil importer countries transfer wealth towards oil exporter countries. As a result of the wealth transfer, the purchasing power of companies and households falls in the oil importer countries. This effect has different implications due to being an oil importer or exporter country. Third, due to the real balance effect [2], the rise in oil prices increases the demand for money. As the monetary authority fails to meet the increase in money demand, interest rates rise which results a reduction in the economic activity. As the monetary authority fails to meet the increase in money demand, an increase in interest rates will cause a fall in economic activity [3]. Fourth, the increase in oil prices will causes inflation which leads to the spiral of price-wage increases. Fifth, it has a negative impact on consumption, investment and stocks. Increasing oil prices leads to a decrease in disposable income and in consumption. Furthermore, investments decrease due to increased costs. Finally, the rise in oil prices reduces employment in the country [4-5] .

Among the emerging economies, Turkey has a significant energy consumption. In the process of economic growth and development, Turkey's hunger for energy has been increasing. Moreover, energy, as a significant input that facilitates economic growth and development, has a strategic importance. However, Turkey does not have the sufficient energy production which results the increase in costs in Turkey, in case of any increase in the price of energy, due to being dependent on external sources.

The main purpose of this study is to examine the dependence structures and/or tail dependence between oil price changes and stock market indices using the Copula-Garch method. In the majority of previous studies, researchers used traditional time series models such as vector autoregression (VAR) and cointegrated vector error-correction (VEC) models [6-7-8-9-10-11-12]. The dependence structure estimated via copulas is more robust since it separates the dependence structure from the choice of margins [13]. The Copula method provides two important advantages in multivariate analysis [14]. Copulas capture nonlinear dependencies and can provide the structure of the dependence and are invariant to increasing and continuous transformations [15]. First, this method allows researchers to separately model marginal distributions and dependencies and their related effects. Second, the copula method captures the dependence between variables completely. There exist a large number of copulas to capture a myriad of dependence structure, allowing fattiredness, asymmetry, tail dependence and so on. The dependence structures among financial markets are important for effective risk management and international asset allocation [16]. 


\section{MATERIAL AND METHOD}

\subsection{Copula Functions}

The copula function is presented to measure dependence of multivariate variables. Based on the famous Sklar's theorem [17], copulas allow to put in place the fruitful idea of splitting the specification of a multivariate model into two parts: the marginal distributions on one side, the dependence structure (copula) on the other part. Let $X$ and $Y$ be random variables with continuous distribution functions $F_{X}$ and $F_{Y}$, which are uniformly distributed on the interval [0,1]. Then, there is a copula such that for all $x, y \in R$,

$$
F_{X Y}(X, Y)=C\left(F_{X}(X), F_{Y}(Y)\right.
$$

The copula $C$ for $(X, Y)$ is the joint distribution function for the pair $F_{X}(X), F_{Y}(Y)$ provided $F_{X}$ and $F_{Y}$ continuous. The copula $C$ for $(X, Y)$ is the joint distribution function for the pair $F_{X}(X), F_{Y}(Y)$ provided $F_{X}$ and $F_{Y}$ continuous.The joint probability density of the variables $X$ and $Y$ is obtained from the copula density $(u, v)=\frac{\partial^{2} C(u, v)}{\partial u \partial v}$, as follows:

$$
f_{x y}(x, y)=c(u, v) f_{x}(\mathrm{x}) f_{y}(y)
$$

where $f_{x}(\mathrm{x})$ and $f_{y}(y)$ are the marginal densities of the random variables $X$ and $Y$. According to Sklar (1959) an n-dimensional joint distribution can be decomposed into its n-univariate marginal distributions and an n-dimensional copula. In the extension of Sklar's theorem to continuous conditional distributions, Patton (2006) shows that the lower (left) and upper (right) tail dependence of two random variables is given for the copula as:

$$
\begin{gathered}
\lambda_{l}=\lim _{u \rightarrow 0} P\left(\mathrm{~F}_{x}(x) \leq u \mid F_{y}(x) \leq u\right)=\lim _{u \rightarrow 0} C(u, u) / u \\
\lambda_{u}=\lim _{u \rightarrow 1} P\left(\mathrm{~F}_{x}(x)>\mathrm{u} \mid F_{y}(x)>u\right)=\lim _{u \rightarrow 1} 1-2 u-C(u, u) / 1-u
\end{gathered}
$$

where $\lambda_{l}$ and $\lambda_{u} \in[0,1]$.

\subsection{Copula Models}

We introduce several copula models in this section; Gumbel copula, Clayton copula, Frank copula Gaussian copula, Student t copula and Joe copula.

Gumbel Copula: This Archimedean copula is defined with the help of generator function $\phi(t)=(-\ln t)^{\theta}, \theta \geq 1$;

$$
C_{\theta}(u, v)=\exp \left(-\left[(-\ln u)^{\theta}+(-\ln v)^{\theta}\right]^{1 / \theta}\right)
$$

where $\theta$ is the copula parameter restricted to $[1, \infty)$. This copula is asymmetric, with more weight in the right tail. Beside this, it is extreme value copula [17].

Clayton Copula: This Archimedean copula is defined with the help of generator function $\phi(t)=\frac{t^{-\theta}-1}{\theta}$,

$$
C_{\theta}(u, v)=\left(u^{-\theta}+v^{-\theta}-1\right) .
$$


where $\theta$ is the copula parameter restricted to $(0, \infty)$. This copula is also asymmetric, but with more weight in the left tail [17].

Frank Copula: This Archimedean copula is defined with the help of generator function; $\phi(t)=-\ln \frac{-e^{-\theta t}-1}{e^{-\theta}-1}$;

$$
C_{\theta}(u, v)=-\frac{1}{\theta} \ln \left(1+\frac{\left(e^{-\theta u}-1\right)\left(e^{-\theta v}-1\right)}{\left(e^{-\theta}-1\right)}\right)
$$

where $\theta$ is the copula parameter restricted to $[-1,1]$.

Joe Copula: This Archimedean copula is defined with the help of generator function; $\phi(t)=-\ln \left[1-(1-t)^{\theta}\right]$

$$
C_{\theta}(u, v)=1-\left[(1-u)^{\theta}+(1-v)^{\theta}-(1-u)^{\theta}(1-v)^{\theta}\right]^{1 / \theta}
$$

where $\theta$ is the copula parameter restricted to $[1, \infty)[17]$.

Gaussian copula: The copula function;

$$
C(u, v ; \rho)=\int_{-\infty}^{\Phi^{-1}(u)} \int_{-\infty}^{\Phi^{-1}(v)} \frac{1}{2 \pi \sqrt{1-\rho^{2}}} \exp \left(\frac{2 \rho r s-r^{2}-s^{2}}{2\left(1-\rho^{2}\right)}\right) d r d s
$$

where $u=F_{Y_{1}}\left(\mathrm{y}_{1}\right), v=F_{Y_{2}}\left(\mathrm{y}_{2}\right)$ is the inverse of the standard normal distribution and $\rho$ is the general correlation coefficient.

Student $t$ copula: This copula allows for joint fat tails and an increased probability of joint extreme events compared with the Gaussian copula. This copula can be written as;

$$
C_{\rho, v}(u, v)=\int_{-\infty}^{t_{\nu}^{-1}(u)} \int_{-\infty}^{t_{\nu}^{-1}(v)} \frac{1}{2 \pi\left(1-\rho^{2}\right)^{1 / 2}}\left\{1+\frac{x^{2}-2 \rho x y+y^{2}}{v\left(1-\rho^{2}\right)}\right\}^{-(v+2) / 2} d s d t
$$

where $\rho, v$ parameters of the $\mathrm{t}$ copula.

The BB1 Copula (Clayton-Gumbel) copula is given by

$$
C(u, v)=1-\left(u_{1}^{-\theta}+u_{2}^{-\theta}-u_{1}^{-\theta} u_{2}^{-\theta}\right)^{1 / \theta}
$$

with $\theta \in[1, \infty)$.

The BB6 Copula (Joe-Gumbel) copula is

$$
C\left(u_{1}, u_{2}, \theta, \delta\right)=1-\left(1-\exp \left\{-\left[\left(-\log \left(1-u_{1}^{-\theta}\right)\right)^{\delta}+\left(-\log \left(1-u_{2}{ }^{-\theta}\right)\right)^{\delta}\right]^{\frac{1}{\delta}}\right\}\right)^{\frac{1}{\theta}}
$$

with $\theta \in[1, \infty) \cap \delta \in[1, \infty)$.

The BB7 (Joe-Clayton) copula is given by

$$
C\left(u_{1}, u_{2}, \theta, \delta\right)=1-\left(1-\left[\left(1-\bar{u}_{1}^{\theta}\right)^{-\delta}+\left(1-\left[\left(1-\bar{u}_{2}^{\theta}\right)^{-\delta}-1\right]^{\frac{1}{\delta}}\right)^{\frac{1}{\theta}}\right.\right.
$$

with $\theta \in[1, \infty) \cap \delta \in[0, \infty)$.

The BB8 (Frank-Joe) copula is

$$
C\left(u_{1}, u_{2}, \theta, \delta\right)=\frac{1}{\delta}\left(1-\left[1-\frac{1}{1-(1-\delta)}\left(1-\left(1-\delta u_{1}\right)^{\theta}\right)\left(1-\left(1-\delta u_{2}\right)^{\theta}\right)\right] \frac{1}{\theta}\right)
$$


with $\theta \in[1, \infty) \cap \delta \in(0,1]$.

\subsection{Marginal Modelling}

In order to build the model for bivariate distribution with the copula, primarily the marginal distribution for the series must be formed. For this, there are models that it has been commonly accepted financial time series returns. ARCH and GARCH model, proposed Engle (1986) and Bollerslev (1986), which have been widely applied Financial series. There are a few GARCH model. In this paper, we combine ARMA $(\mathrm{m}, \mathrm{n})$ and $\mathrm{ARCH}(\mathrm{p}, \mathrm{q}), \mathrm{GARCH}(1,1)$, EGARCH nad GJRGARCH (p,q) model to daily financial returns. This models;

$$
\begin{gathered}
r_{t}=\lambda_{0}+\sum_{j=1}^{m} \lambda_{j} r_{t-j}+\varepsilon_{t}-\sum_{i=1}^{n} \theta_{i} \varepsilon_{t-i} \\
r_{t}=w_{0}+\sum_{i=1}^{q} \beta_{i} u_{t-1}^{2} \\
r_{t}=w_{0}+\sum_{i=1}^{q} \alpha_{i} u_{t-1}^{2}+\sum_{j=1}^{p} \beta_{j} u_{t-1}^{2} \\
\log \left(r_{t}\right)=w_{0}+\sum_{i=1}^{q} \alpha_{i} \frac{\left|u_{t-i}\right|}{\sqrt{r_{t-i}}}+\sum_{i=1}^{q} \gamma_{i} \frac{u_{t-i}}{\sqrt{r_{t-i}}}+\sum_{j=1}^{p} \beta_{j} \log \left(u_{t-j}\right) \\
r_{t}=w_{0}+\sum_{i=1}^{p} \beta_{i} r_{t-i}+\sum_{j=1}^{q} \alpha_{j} u_{t-j}^{2}+\sum_{i=1}^{q} \gamma_{j} u_{t-j}^{2} I_{t-j}
\end{gathered}
$$

where m,n,p, q are positive integers , $u_{t}=\eta_{t} \sqrt{h_{t}}, \eta_{t} \square f(0,1)$, respectively $\lambda_{j}, \theta_{i}$ parameters of (AR) and (MA), $w_{0}, \beta_{i}, \alpha_{j}, \gamma_{j}$ are ARCH(p,q), GARCH (1,1), EGARCH nad GJR-GARCH (p,q) model parameters.

\section{DATA}

XU030 data was downloaded from www.investing.com. as daily prices between 02.01.2003 - 13.04.2017. Table 1 submit the stochastic properties of financial series over the period (2003-2017) and Table 2 submit the stochastic properties of the daily returns of the XU030, Gold Spot, Brent Oil and Natural Gas over the period (2003-2017). This table indicate the positive average return series for Gold Spot and Brent Oil, the negative average return series for XU030 and Natural Gas. Among all the series, Natural Gas is the most volatile as indicated by the standard deviation. The positive values for skewness are extensive for all the series with the exception of the Natural Gas. The kurtosis value is greater than three, indicating the presence of a fat tailed distribution for all the returns series. In addition, the Jarque-Bera test rejects the null of Gaussian distribution for all the series. By applying the two-unit root tests (ADF and PP), the stationary test (KPSS), we find that all return series are stationary. Finally, the Autoregressive Conditional Heteroscedasticity -Lagrange Multiplier (ARCH-LM) test indicates that there exist strong ARCH effects in all the four financial return series.

Table 1. Summary Statistics

\begin{tabular}{lcccc}
\hline & XU030 & Gold Spot & Brent Oil & Natural gas \\
\hline Mean & 70502,58 & 1010,029 & 71,68646 & 5060,293 \\
Median & 71042,27 & 1125,250 & 65,26000 & 4405,000 \\
Maksimum & 147935,8 & 1898,100 & 143,9500 & 15378,00 \\
Minumum & 12951,90 & 323,0000 & 23,23000 & 1639,000 \\
Std.Dev & 29767,28 & 418,0472 & 28,89175 & 2360,924 \\
Skewness & 0,131419 & $-0,108938$ & 0,306036 & 1,324039 \\
Kurtosis & 2,352783 & 1,870957 & 1,877946 & 5,052819 \\
Jarqure Bera & 74,92431 & 203,0140 & 250,8313 & 1723,718 \\
Probability & 0,00000 & 0,000000 & 0,00000 & 0,00000
\end{tabular}


Table 2. Summary Statistics of return series

\begin{tabular}{ccccc}
\hline & XU030 & Gold Spot & Brent Oil & Natural gas \\
\hline Mean & $-0,000653$ & 0,000368 & 0,000228 & $-0,000148$ \\
Median & $-0,000860$ & 0,000588 & 0,000354 & $-0,000757$ \\
Maksimum & 0,109019 & 0,104371 & 0,181297 & 0,324354 \\
Minumum & $-0,127255$ & $-0,088756$ & $-0,168320$ & $-0,198993$ \\
Std.Dev & 0,018059 & 0,011856 & 0,021999 & 0,032895 \\
Skewness & 0,062663 & 0,326240 & 0,071758 & $-0,777334$ \\
Kurtosis & 6,407444 & 9,272974 & 7,713053 & 9,731479 \\
Jarqure Bera & 1784,650 & 6105,605 & 3412,836 & 7326,525 \\
Probability & 0.000000 & 0.000000 & 0.000000 & 0.000000 \\
ARCH LM & 58,67665 & 145,3111 & 39,77587 & 17,67795 \\
ADF & $-61,07679$ & $-60,05891$ & $-58,25491$ & $-61,46372$ \\
PP & $-61,08128$ & $-60,05663$ & $-58,24363$ & $-61,48292$ \\
KPSS & 0,052393 & 0,159194 & 0,153248 & 0,037679 \\
Q(20) & 7,0920 & 7,2645 & 7,1419 & 6,8666 \\
No. Of Obs & 3685 & 3685 & 3685 & 3685
\end{tabular}
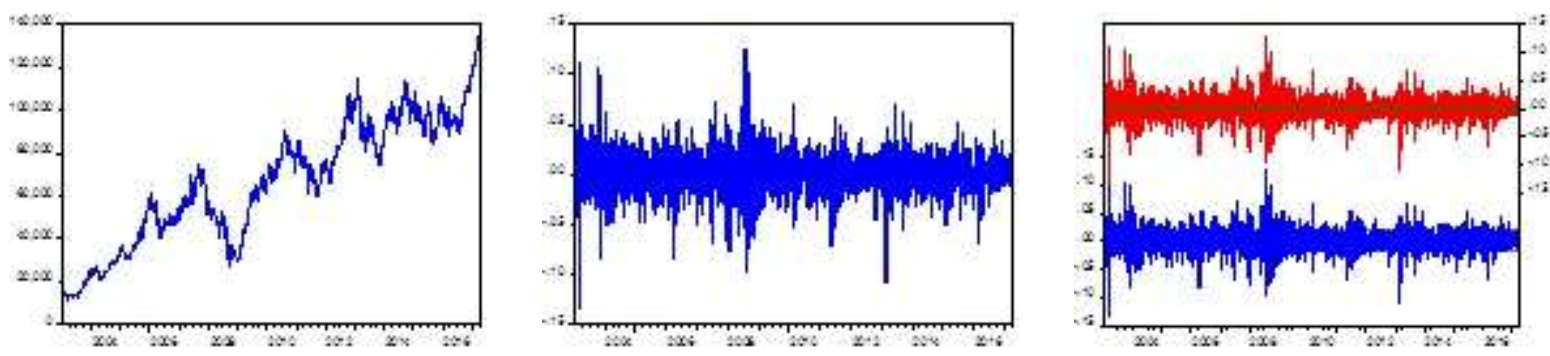

Figure 1: Change over years of XU030 series and XU030 return series and Residual graphs
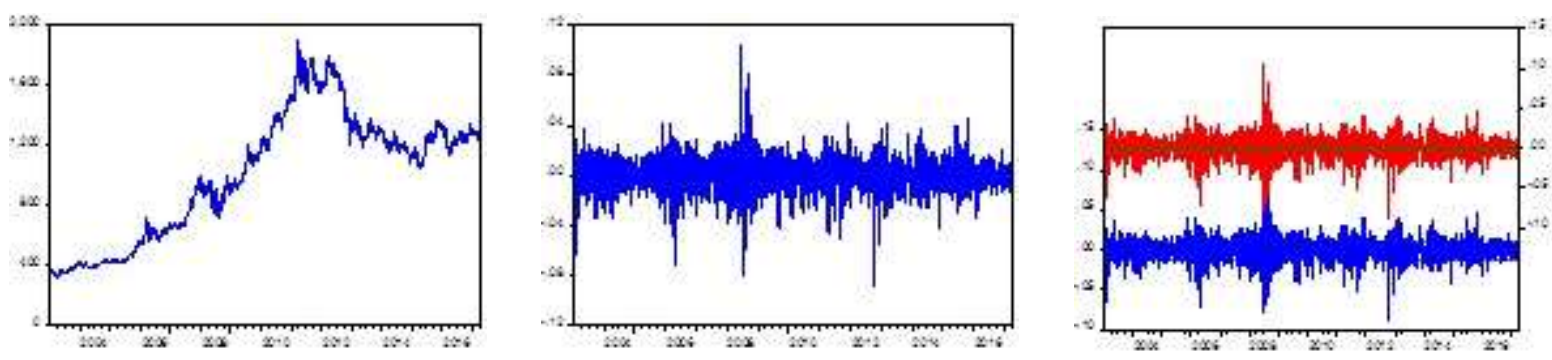

Figure 2: Change over years of Gold Spot series and Gold Spot return series and Residual graphs
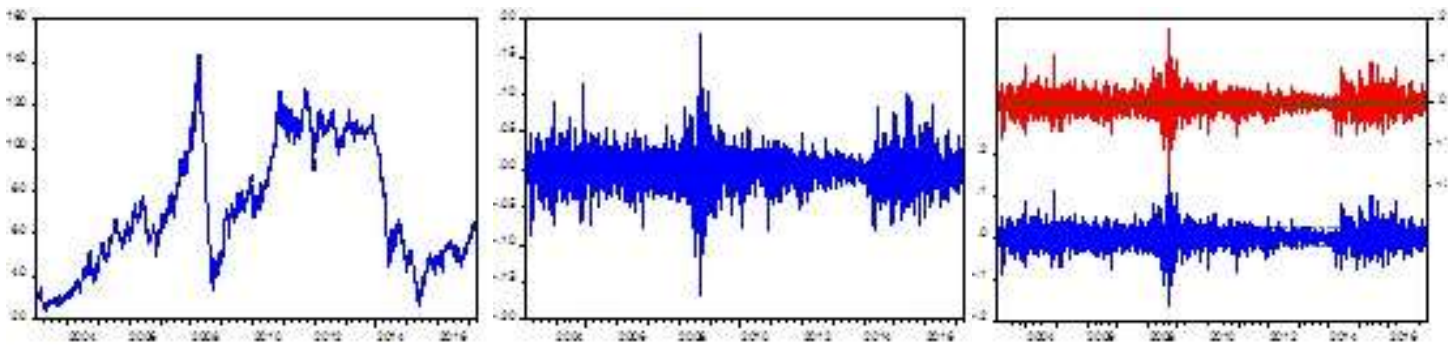

Figure 3: Change over years of Brent Oil series and Brent Oil return series and Residual graphs 

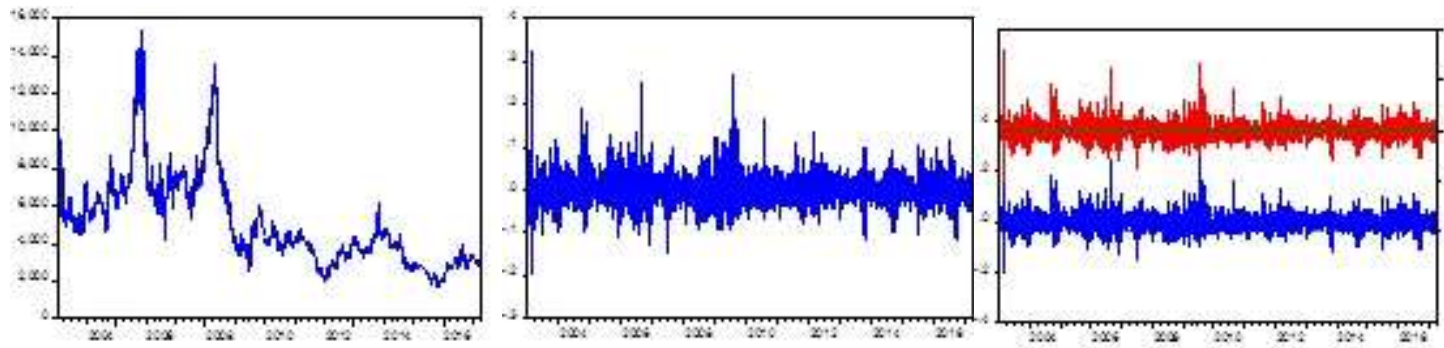

Figure 4: Change over years of Natural gas series and Natural gas return series and Residual graphs

\subsection{Results of marginal distrubution}

In this paper, the ARMA (m,n) GARCH models for financial return series are used.We choose the most suitable with the help of criteria AIC, SIC and HQIC. Table. All the parameters estimate of marginal distributions are included in Table 3 and Table 4 which summarize the best model for all the four marginal distributions: the best models for the marginal; XU030 Gold Spot, Brent Oil and Natural gas; ARMA (3,2)-EGARCH $(1,1,1)$ student t, ARMA (4,4)-GJRGARCH $(1,1)$ student t, ARMA $(4,4)$-EGARCH $(1,1,1)$ student t, ARMA $(4,4)$-EGARCH $(1,1,1)$ student $t$ respectively. Here, the EGARCH model has been chosen to take into account the asymmetric effect. it is seen that $\gamma$ parameter, which has asymmetrical effect, is positive statistically significant for natural gas return series and for the XU030 and Brent Oil return series is negative. Namely, from table 4, XU030 and Brent Oil have leverage affect, XU030 and Brent Oil have a negative correlation between the past and the future volatility of returns. This state, it shows that for XU030 and Brent Oil asymmetrical effect are stated that according to good news of bad news, the stock has further increased its return volatility. From table 4, Gold Spot has ARMA $(4,4)-G J R-G A R C H ~(1,1)$, namely this model consider that effect of negative shocks and positive shocks is not symmetrical. In table 4, for Gold Spot return series, $\gamma<0$, it show that the effect on volatility of positive news indicates more than the effect of negative news. According to table 4 Results of ARCH-LM test (Table 4) show that XU030, Brent Oil and Natural Gas are neither autocorrelation nor ARCH effects exist in the residuals but, in table 4, for Gold Spot, it is seen that the variance problem and the ARCH effect are not completely solved.

Table 3. Mean Equation

\begin{tabular}{ccccc} 
& XU030 & Gold Spot & Brent Oil & Natural gas \\
\hline Mean Equation & $\mathbf{( 4 , 4 )}$ & $\mathbf{( 4 , 4 )}$ & $\mathbf{( 4 , 4 )}$ & $\mathbf{( 4 , 4 )}$ \\
\hline$\lambda_{1}$ & 0,091393 & $-1,278051$ & $-1,091093$ & $-0,511633$ \\
$\lambda_{2}$ & 1,084073 & $-0,832286$ & $-0,354479$ & $-00,17724$ \\
$\lambda_{3}$ & $-0,055822$ & $-1,238166$ & $-1,083223$ & $-0,545121$ \\
$\lambda_{4}$ & $-0,876281$ & $-0,909126$ & $-0,972216$ & $-0,812788$ \\
$\boldsymbol{\theta}_{1}$ & $-0,103965$ & 1,277735 & 1,091562 & $-0,517096$ \\
$\boldsymbol{\theta}_{2}$ & $-1,089174$ & 0,808185 & 0,359669 & 0,207752 \\
$\boldsymbol{\theta}_{3}$ & 0,063884 & 1,230222 & 1,105343 & 0,512102 \\
$\boldsymbol{\theta}_{4}$ & 0,847465 & 0,926261 & 0,983075 & 0,809544 \\
$\mathrm{AIC}$ & $-5,12471$ & $-6,033586$ & $-4,798550$ & 13,38525 \\
SIC & $-5,110613$ & $-6,016725$ & $-4,781688$ & 13,40211 \\
$\mathrm{HQIC}$ & $-5,121470$ & $-6,027584$ & $-4,792548$ & 13,39125 \\
\hline
\end{tabular}


Table 4. Variance Equation

\begin{tabular}{|c|c|c|c|c|c|c|c|c|}
\hline $\begin{array}{l}\text { Variance } \\
\text { Equation }\end{array}$ & XU030 & & $\begin{array}{l}\text { Gold } \\
\text { Spot }\end{array}$ & & $\begin{array}{c}\text { Brent } \\
\text { Oil }\end{array}$ & & $\begin{array}{c}\text { Natural } \\
\text { gas }\end{array}$ & \\
\hline $\operatorname{ARCH}(1,0)$ & Gaussian & $\begin{array}{c}\text { Student } \\
\mathbf{t}\end{array}$ & Gaussian & Student $\mathbf{t}$ & Gaussian & $\begin{array}{c}\text { Student } \\
t\end{array}$ & Gaussian & Student $\mathbf{t}$ \\
\hline$w_{0}$ & 0,000293 & 0,000291 & 0,000120 & 0,000127 & 0,000416 & 0,000424 & 0,000871 & 0,000944 \\
\hline$\beta$ & 0,156081 & 0,171638 & 0,151878 & 0,141077 & 0,281357 & 0,154450 & 0,212928 & 0,119735 \\
\hline AIC & $\begin{array}{c}- \\
5,159615\end{array}$ & $\begin{array}{c}- \\
5,253597\end{array}$ & $\begin{array}{c}- \\
6,062482\end{array}$ & $-6,203461$ & $\begin{array}{c}- \\
4,941518\end{array}$ & $-4,938634$ & $\begin{array}{c}- \\
4,027482\end{array}$ & $-4,129189$ \\
\hline SIC & $\begin{array}{c}- \\
5,154557\end{array}$ & $\begin{array}{c}- \\
5,246853\end{array}$ & $\begin{array}{c}- \\
6,057423\end{array}$ & $-6,196716$ & $\begin{array}{c}- \\
4,937201\end{array}$ & $-4,931889$ & $\begin{array}{c}- \\
4,022424\end{array}$ & $-4,122444$ \\
\hline ARCH LM & 0,009111 & 0,175994 & 0,137327 & 0,000770 & 0,015181 & 0,203494 & 0,585391 & 0,591041 \\
\hline Probability & 9240 & 0,6748 & 0,7110 & 0,9779 & 0,9019 & 0,6519 & 0,4442 & 0,4420 \\
\hline $\operatorname{GARCH}(1,1)$ & Gaussian & $\begin{array}{c}\text { Student } \\
\mathbf{t}\end{array}$ & Gaussian & Student $\mathbf{t}$ & Gaussian & $\begin{array}{c}\text { Student } \\
t\end{array}$ & Gaussian & Student $\mathbf{t}$ \\
\hline$w_{0}$ & $5,78 \mathrm{E}-06$ & $4,44 \mathrm{E}-06$ & $1,12 \mathrm{E}-06$ & $9,27 \mathrm{E}-07$ & $1,11 \mathrm{E}-06$ & $1,12 \mathrm{E}-06$ & $1,56 \mathrm{E}-05$ & $2,01 \mathrm{E}-015$ \\
\hline$\alpha$ & 0,071282 & 0,062744 & 0,041681 & 0,037078 & 0,042299 & 0,039089 & 0,066405 & 0,059656 \\
\hline$\beta$ & 0,912450 & 0,924535 & 0,950134 & 0,956521 & 0,956190 & 0,959349 & 0,921375 & 0,920844 \\
\hline AIC & $\begin{array}{c}- \\
5,293915\end{array}$ & $\begin{array}{c}- \\
5,339525\end{array}$ & $\begin{array}{c}- \\
6,213366\end{array}$ & $-6,28$ & 5,014436 & 5,048675 & 4,128564 & , 197193 \\
\hline SIC & $\begin{array}{c}- \\
5,287170\end{array}$ & $\begin{array}{c}- \\
5,331094\end{array}$ & $\begin{array}{c}- \\
6,206621\end{array}$ & $-6,277709$ & 5,007691 & 5,04 & $\begin{array}{c}- \\
4,121819\end{array}$ & $-4,188762$ \\
\hline ARCH LM & 0,527507 & 1,343747 & 10,33822 & 15,84413 & 0,239000 & 0,090033 & 0,073371 & 0,192731 \\
\hline Probability & 0,4677 & 0,2464 & 0,0013 & 0,0001 & 0,6249 & 0,7641 & 0,7865 & 0,6607 \\
\hline $\operatorname{EGARCH}(\mathbf{1}, \mathbf{0 , 1})$ & Gaussian & $\begin{array}{c}\text { Student } \\
\mathbf{t}\end{array}$ & Gaussian & Student $\mathbf{t}$ & Gaussian & $\begin{array}{c}\text { Student } \\
\mathbf{t}\end{array}$ & Gaussian & Student $\mathbf{t}$ \\
\hline$w_{0}$ & $\begin{array}{c}- \\
8,161278\end{array}$ & $\begin{array}{c}- \\
8,157195\end{array}$ & $\begin{array}{c}- \\
9,075447\end{array}$ & $-9,005908$ & $\begin{array}{c}- \\
7,827831\end{array}$ & $\begin{array}{c}- \\
7,800080\end{array}$ & $\begin{array}{c}- \\
7,105400\end{array}$ & $\begin{array}{c}- \\
6,9997761\end{array}$ \\
\hline$\alpha$ & 0,225078 & 0,229057 & 0,245213 & 0,214887 & 0,230518 & 0,239340 & 0,331653 & 0,195122 \\
\hline$\gamma$ & $\begin{array}{c}- \\
0,102033\end{array}$ & $\begin{array}{c}- \\
0,127242\end{array}$ & $\begin{array}{c}- \\
0,087383\end{array}$ & $-0,070960$ & $\begin{array}{c}- \\
0,061995\end{array}$ & $\begin{array}{c}- \\
0,102007\end{array}$ & $\begin{array}{c}- \\
0,055778\end{array}$ & $-0,027502$ \\
\hline AIC & $\begin{array}{c}- \\
5,156514\end{array}$ & $\begin{array}{c}- \\
5,252132\end{array}$ & $\begin{array}{c}- \\
6,060198\end{array}$ & $-6,199981$ & $\begin{array}{c}- \\
4,821720\end{array}$ & $\begin{array}{c}- \\
4,936032\end{array}$ & $\begin{array}{c}- \\
4,022348\end{array}$ & $-4,126759$ \\
\hline SIC & & 5,24 & 6,053 & $-6,191550$ & 4,81 & 4,92 & 4,015604 & $-4,118328$ \\
\hline ARCH LM & 1,098925 & 0,627135 & 0,520245 & 1,820095 & 2,430359 & 2,676269 & 0,122077 & 3,681254 \\
\hline Probability & 0,2945 & 0,4284 & 0,4707 & 0,1773 & 0,1190 & 0,1019 & 0,7268 & 0,0550 \\
\hline $\operatorname{EGARCH}(0,1,1)$ & Gaussian & $\begin{array}{c}\text { Student } \\
\mathbf{t}\end{array}$ & Gaussian & Student $\mathbf{t}$ & Gaussian & $\begin{array}{c}\text { Student } \\
\mathbf{t}\end{array}$ & Gaussian & Student $\mathbf{t}$ \\
\hline$w_{0}$ & $\stackrel{-}{0,490530}$ & $\begin{array}{c}- \\
0,573489\end{array}$ & $\begin{array}{c}- \\
4,115993\end{array}$ & $-4,591960$ & $\begin{array}{c}- \\
0,079891\end{array}$ & $-0,038480$ & $\begin{array}{c}- \\
8,923357\end{array}$ & $-10,28621$ \\
\hline$\gamma$ & $\begin{array}{c}- \\
0,077465\end{array}$ & $\begin{array}{c}- \\
0,094684\end{array}$ & $\begin{array}{c}- \\
0,083775\end{array}$ & $-0,090243$ & $\begin{array}{c}- \\
0,044884\end{array}$ & $-0,029277$ & $\begin{array}{c}- \\
0,028191\end{array}$ & $-0,017373$ \\
\hline$\beta$ & 0,938911 & 0,928653 & 0,536373 & 0,480277 & 0,989760 & 0,995098 & 0,306515 & $-0,504465$ \\
\hline AIC & $\begin{array}{c}- \\
5,178566\end{array}$ & $-5,26473$ & $\begin{array}{c}- \\
6,037769\end{array}$ & $-6,192302$ & $\begin{array}{c}- \\
4,891704\end{array}$ & $-4,955395$ & $\begin{array}{c}- \\
3,989809\end{array}$ & $-4,120062$ \\
\hline SIC & $\begin{array}{c}- \\
5,171165\end{array}$ & $-5,25630$ & $\begin{array}{c}- \\
6,031051\end{array}$ & $-6,183871$ & $\begin{array}{c}- \\
4,884959\end{array}$ & $-4,946964$ & $\begin{array}{c}- \\
3,983064\end{array}$ & $-4,111631$ \\
\hline $\begin{array}{l}\text { ARCH LM } \\
\text { Probability }\end{array}$ & $\begin{array}{c}43,82563 \\
0,0000\end{array}$ & $\begin{array}{c}38,09718 \\
0,0000\end{array}$ & $\begin{array}{c}36,62184 \\
0,00000\end{array}$ & $\begin{array}{c}36,81364 \\
0,0000\end{array}$ & $\begin{array}{c}20,03207 \\
0,00000\end{array}$ & $\begin{array}{c}29,69820 \\
0,00000\end{array}$ & $\begin{array}{c}19,15973 \\
0,0000\end{array}$ & $\begin{array}{c}18,26494 \\
0,0000\end{array}$ \\
\hline $\operatorname{EGARCH}(\mathbf{1}, \mathbf{1 , 1})$ & Gaussian & $\begin{array}{c}\text { Student } \\
t\end{array}$ & Gaussian & Student $\mathbf{t}$ & Gaussian & $\begin{array}{c}\text { Student } \\
\mathbf{t}\end{array}$ & Gaussian & Student $\mathrm{t}$ \\
\hline$w_{0}$ & 0,320760 & 0,295327 & 0,164738 & $-0,126331$ & 7,633746 & $-0,086584$ & 0,215018 & $-0,238740$ \\
\hline$\alpha$ & 0,153358 & 0,143329 & 0,106468 & 0,090762 & 0,010000 & 0,076884 & 0,146041 & 0,137357 \\
\hline
\end{tabular}




\begin{tabular}{|c|c|c|c|c|c|c|c|c|}
\hline$\gamma$ & $\begin{array}{c}- \\
0,052580\end{array}$ & $-0,05370$ & 0,001761 & 0,025767 & 0,010000 & $-0,038812$ & 0,003331 & 0,009183 \\
\hline$\beta$ & 0,975044 & 0,97732 & 0,990527 & 0,993394 & 0,010000 & 0,996479 & 0,984986 & 0,980816 \\
\hline AIC & $\begin{array}{c}- \\
5,299467\end{array}$ & $-5,34419$ & $\begin{array}{c}- \\
6,207597\end{array}$ & $\begin{array}{c}- \\
6,2880079\end{array}$ & $\begin{array}{c}- \\
4,792335\end{array}$ & $-5,055727$ & $\begin{array}{c}- \\
4,132234\end{array}$ & $-4,199140$ \\
\hline SIC & $\begin{array}{c}- \\
5,291037\end{array}$ & $-5,33407$ & $\begin{array}{c}- \\
6,199166\end{array}$ & $-6,277962$ & $\begin{array}{c}- \\
4,783905\end{array}$ & $-5,045610$ & $\begin{array}{c}- \\
4,123804\end{array}$ & $-4,189023$ \\
\hline ARCH LM & 0,335602 & 0,60967 & 22,85389 & 60,99098 & 133,4673 & 0,000350 & 0,048564 & 0,098474 \\
\hline Probability & 0,5624 & 0,4349 & 0,00000 & 0,00000 & 0,00000 & 0,9851 & 0,8256 & 0,7537 \\
\hline GJR-GARCH & Gaussian & $\begin{array}{c}\text { Student } \\
\mathbf{t}\end{array}$ & Gaussian & Student $\mathbf{t}$ & Gaussian & Student $\mathbf{t}$ & Gaussian & Student $\mathbf{t}$ \\
\hline$w_{0}$ & $7,57 \mathrm{E}-06$ & $6,61 \mathrm{E}-06$ & $1,08 \mathrm{E}-06$ & $8,07 \mathrm{E}-07$ & $7,46 \mathrm{E}-07$ & $6,49 \mathrm{E}-07$ & $1,57 \mathrm{E}-05$ & $2,05 \mathrm{E}-05$ \\
\hline$\alpha$ & 0,042067 & 0,003833 & 0,050134 & 0,55406 & 0,009428 & 0,011518 & 0,066787 & 0,063294 \\
\hline$\gamma$ & 0,066152 & 0,062373 & $\begin{array}{c}- \\
0,015708\end{array}$ & $-0,034584$ & 0,051649 & 0,043492 & $\begin{array}{c}- \\
0,000892\end{array}$ & $-0,00818$ \\
\hline$\beta$ & 0,902220 & 0,910036 & 0,950414 & 0,958233 & 0,963833 & 0,965510 & 0,921371 & 0,920542 \\
\hline AIC & $\begin{array}{c}- \\
5,301613\end{array}$ & $\begin{array}{c}- \\
5,344441\end{array}$ & $\begin{array}{c}- \\
6,214008\end{array}$ & $-6,289445$ & $\begin{array}{c}- \\
5,029293\end{array}$ & $-5,055483$ & $\begin{array}{c}- \\
4,128023\end{array}$ & $-4,196735$ \\
\hline SIC & $\begin{array}{c}- \\
5,293182\end{array}$ & $\begin{array}{c}- \\
5,333432\end{array}$ & $\begin{array}{c}- \\
6,205577\end{array}$ & $-6,279328$ & $\begin{array}{c}- \\
5,020862\end{array}$ & $-5,045367$ & $\begin{array}{c}- \\
4,119592\end{array}$ & $-4,186618$ \\
\hline ARCH LM & 0.260751 & 0.674007 & 15,28889 & 36,46513 & 0,177160 & 0,108969 & 0,070893 & 0,156678 \\
\hline Probability & 0,129960 & 0,014129 & 0,0001 & 0,00000 & 0,6738 & 0,7413 & 0,7900 & 0,6922 \\
\hline
\end{tabular}

\subsection{Results for the copula models}

In this study, to model dependence of XU030- Gold Spot, XU030- Brent Oil and XU030-Natural Gas pairs, we use copula family in table 5, table 6 and table 7. From Table 5,6 and 7, it is obvious that the for C, XU030- Brent Oil and XU030-Natural Gas pairs, Survival BB8, Rotated Tawn Type 2180 degrees and Rotated BB8 270 degrees copula best performs according to the AIC, BIC criteria respectively. The obtained tail dependence values for the pairs, while XU030Gold Spot and B XU030-Natural Gas have symmetric tail dependency, XU030- Brent Oil pair has $\lambda_{u}=0$ and $\lambda_{l}=0,42$ namely for distribution of this pairs has lower tail dependence.
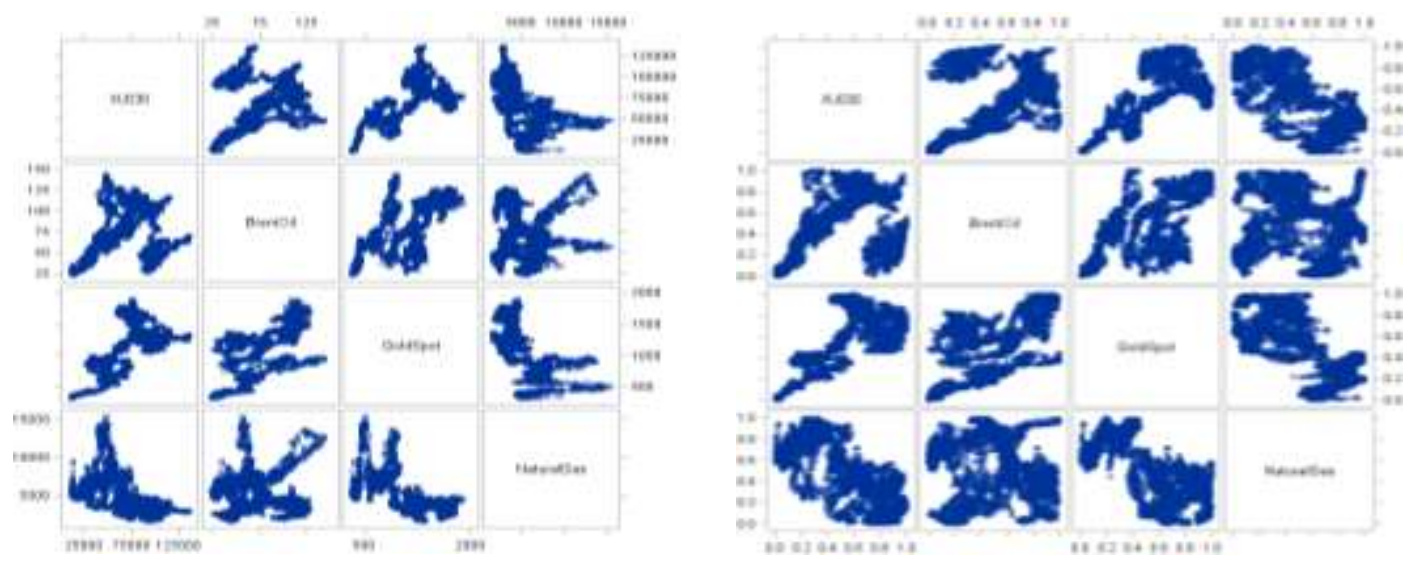

Figure 6. For XU030- Gold Spot, Brent Oil and Natural Gas Raw Data and Transformed Data Scatter Graph 

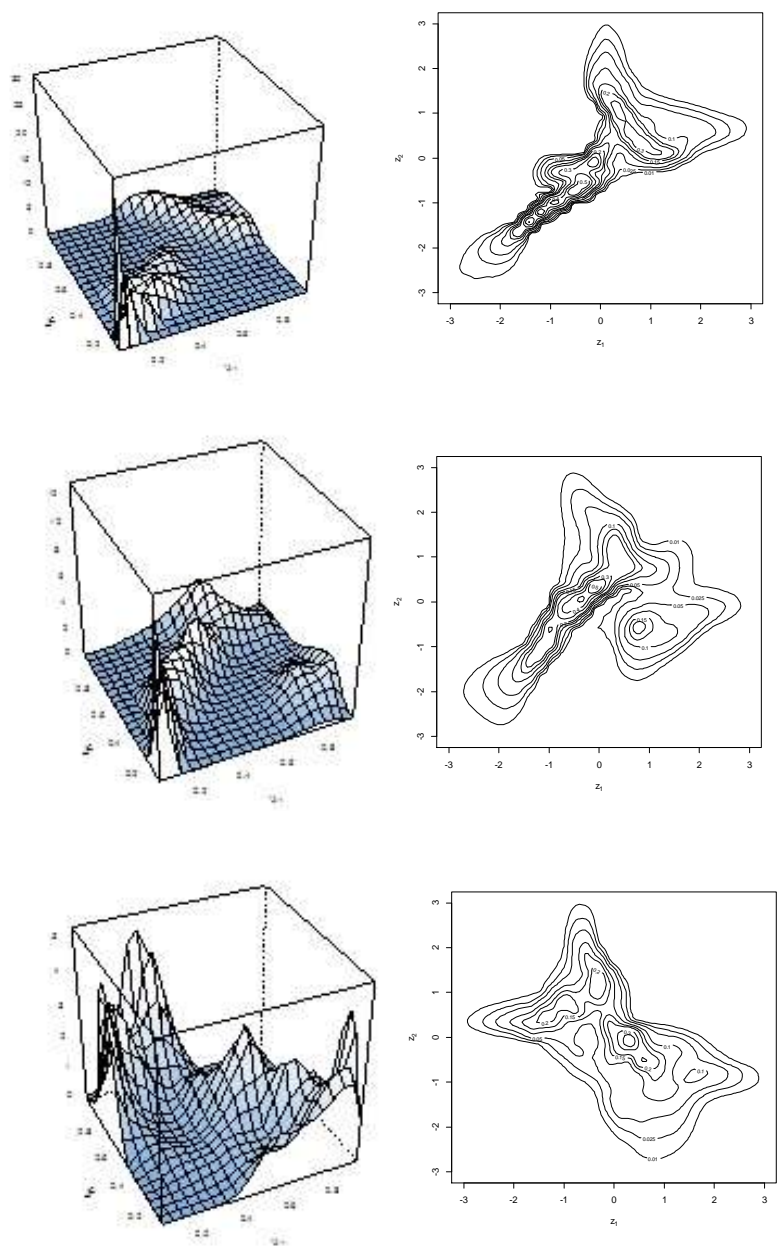

Figure 7. For XU030- Gold Spot, XU030- Brent Oil and XU030-Natural Gas pairs Kernel distribution estimation function graphs.

Table 5. XU030-Gold Spot

\begin{tabular}{|c|c|c|c|c|c|c|c|c|}
\hline Family & $\theta$ & $\rho$ & $V$ & $\lambda_{u}$ & $\lambda_{l}$ & $\log L$ & AIC & BIC \\
\hline \multicolumn{9}{|l|}{ Gaussian } \\
\hline Student $\mathbf{t}$ & - & 0,72 & 6,88 & 0,29 & 0,29 & 1240,53 & $-2477,05$ & $-2464,63$ \\
\hline Clayton & 3,08 & & & 0 & 0,8 & 2356,04 & $-4710,08$ & $-4703,87$ \\
\hline Gumbel & 1,66 & & & 0,48 & 0 & 646,23 & $-1290,46$ & $-1284,25$ \\
\hline Frank & 6,71 & & & 0 & 0 & 1415,47 & $-2828,93$ & $-2822,72$ \\
\hline Joe & 1,48 & & & 0,4 & 0 & 154,91 & $-307,82$ & $-301,6$ \\
\hline BB1 & & 3,08 & 1 & 0 & 0,8 & 2355,18 & $-4706,36$ & 4693,94 \\
\hline BB6 & & 1 & 1,65 & 0,48 & 0 & 645,44 & $-1286,89$ & $-1274,47$ \\
\hline BB7 & & 1 & 3,08 & 0 & 0,8 & 2355,68 & $-4707,37$ & $-4694,94$ \\
\hline BB8 & & 6 & 0,57 & 0 & 0 & 1018,83 & $-2033,65$ & 2021,23 \\
\hline Survival Clayton & 0,62 & & & 0,33 & 0 & 295,88 & $-589,75$ & $-583,54$ \\
\hline Survival Gumbel & 2,33 & & & 0 & 0,65 & 1826,11 & $-3650,21$ & -3644 \\
\hline Survival Joe & 3,84 & & & 0 & 0,8 & 2392,48 & $-4782,95$ & $-4776,74$ \\
\hline Survival BB1 & & 0 & 2,33 & 0 & 0,65 & 1824,83 & $-3645,66$ & $-3633,24$ \\
\hline Survival BB6 & & 3,84 & 1 & 0 & 0,8 & 2392,04 & $-4780,08$ & $-4767,66$ \\
\hline Survival BB7 & & 3,84 & 0 & 0 & 0,8 & 2392 & $-4779,99$ & $-4767,57$ \\
\hline Survival BB8 & & 4,18 & 0,99 & $\mathbf{0}$ & 0 & 2470,79 & $-4937,59$ & 4925,16 \\
\hline Tawn type 1 & & 1,7 & 0,74 & 0,42 & 0 & 546,59 & $-1089,18$ & $-1076,76$ \\
\hline Rotated Tawn Type 1180 degrees & & 2,88 & 0,74 & 0 & 0,61 & 1829 & $-3655,56$ & $-3643,13$ \\
\hline Tawn type 2 & & 1,92 & 0,74 & 0,48 & 0 & 680,24 & $-1356,49$ & $-1344,07$ \\
\hline Rotated Tawn Type 2180 degrees & & 2,47 & 0,74 & 0 & 0,57 & 1638,03 & $-3272,06$ & 3259,64 \\
\hline
\end{tabular}


Table 6. XU030-Brent Oil

\begin{tabular}{|c|c|c|c|c|c|c|c|c|}
\hline Family & $\theta$ & $\rho$ & $V$ & $\lambda_{u}$ & $\lambda_{l}$ & $\log L$ & AIC & BIC \\
\hline \multicolumn{9}{|l|}{ Gaussian } \\
\hline Student $\mathbf{t}$ & & 0,36 & & 0 & 0 & 256,84 & $-511,68$ & $-505,47$ \\
\hline Clayton & - & 0,37 & 4,48 & 0,17 & 0,17 & 301,77 & $-599,54$ & $-587,11$ \\
\hline Gumbel & 1,02 & & & 0 & 0,51 & 719,64 & $-1437,27$ & $-1431,06$ \\
\hline Frank & 1,17 & & & 0,19 & 0 & 66,83 & $-131,67$ & $-125,46$ \\
\hline Joe & 2,2 & & & 0 & 0 & 219,01 & $-436,01$ & $-429,8$ \\
\hline BB1 & 1 & & & 0 & 0 & $-0,02$ & 2,05 & 8,26 \\
\hline BB6 & & 1,01 & 1 & 0 & 0,51 & 718,59 & $-1433,17$ & $-1420,75$ \\
\hline BB7 & & 1 & 1,7 & 0,19 & 0 & 66,21 & $-128,41$ & $-115,99$ \\
\hline BB8 & & 1 & 1,02 & 0 & 0,51 & 719,01 & $-1434,02$ & $-1421,59$ \\
\hline Survival Clayton & & 6 & 0,28 & 0 & 0 & 167,17 & $-330,33$ & $-317,91$ \\
\hline Survival Gumbel & 0,03 & & & 0 & 0 & 0,57 & 0,87 & 7,08 \\
\hline Survival Joe & 1,43 & & & 0 & 0,38 & 554,83 & $-1107,65$ & $-1101,44$ \\
\hline Survival BB1 & 1,88 & & & 0 & 0,55 & 789,09 & $-1576,18$ & $-1569,97$ \\
\hline Survival BB6 & & 0 & 1,43 & 0 & 0,37 & 553,88 & $-1103,75$ & $-1091,33$ \\
\hline Survival BB7 & & 1,88 & 1 & 0 & 0,55 & 788,6 & $-1573,21$ & $-1560,78$ \\
\hline Survival BB8 & & 1,88 & 0 & 0 & 0,55 & 788,42 & $-1572,84$ & $-1560,42$ \\
\hline Tawn type 1 & & 1,93 & 1 & 0 & 0 & 799,69 & $-1595,38$ & $-1582,96$ \\
\hline Rotated Tawn Type 1180 degrees & & 1,67 & 0,41 & 0,28 & 0 & 152,86 & $-301,73$ & $-289,3$ \\
\hline Tawn type 2 & & 1,13 & 0,47 & 0,1 & 0 & 26,58 & $-49,17$ & $-36,74$ \\
\hline Rotated Tawn Type 2180 degrees & & 2,75 & 0,47 & $\mathbf{0}$ & $\mathbf{0 , 4 2}$ & 867,2 & $-1730,4$ & $-1717,97$ \\
\hline
\end{tabular}

Table 7. XU030-Natural Gas

\begin{tabular}{|c|c|c|c|c|c|c|c|c|}
\hline Family & $\theta$ & $\rho$ & $\boldsymbol{V}$ & $\lambda_{u}$ & $\lambda_{l}$ & $\log L$ & AIC & BIC \\
\hline Gaussian & & $-0,58$ & & 0 & 0 & 757,22 & $-1512,44$ & $-1506,23$ \\
\hline Student $\mathrm{t}$ & - & $-0,58$ & 30 & 0 & 0 & 719,74 & $-1435,47$ & $-1423,05$ \\
\hline Frank & $-4,95$ & & & 0 & 0 & 986,94 & $-1971,88$ & $-1965,67$ \\
\hline Rotated Clayton 90 degrees & $-0,87$ & & & 0 & 0 & 589,36 & $-1176,72$ & $-1170,51$ \\
\hline Rotated Gumbel 90 degrees & $-1,48$ & & & 0 & 0 & 444,58 & $-887,15$ & $-880,94$ \\
\hline Rotated Joe 90 degrees & $-1,5$ & & & 0 & 0 & 195,52 & $-389,05$ & $-382,84$ \\
\hline Rotated BB1 90 degrees & & $-0,79$ & 1,05 & 0 & 0 & 590,21 & $-1176,42$ & $-1163,99$ \\
\hline Rotated BB6 90 degrees & & -1 & 1,48 & 0 & 0 & 444,15 & $-884,29$ & $-871,87$ \\
\hline Rotated BB7 90 degrees & & -1 & 0,87 & 0 & 0 & 589,12 & $-1174,25$ & $-1161,82$ \\
\hline Rotated BB8 90 degrees & & -6 & 0,54 & 0 & 0 & 862,6 & $-1721,22$ & $-1708,79$ \\
\hline Rotated Clayton 270 degrees & $-0,63$ & & & 0 & 0 & 339,11 & $-676,21$ & -670 \\
\hline Rotated Gumbel 270 degrees & $-1,56$ & & & 0 & 0 & 618,77 & $-1235,55$ & $-1229,34$ \\
\hline Rotated Joe 270 degrees & $-1,77$ & & & 0 & 0 & 488,19 & $-974,38$ & $-968,17$ \\
\hline Rotated BB1 270 degrees & & 0 & 1,56 & 0 & 0 & 618,64 & $-1233,27$ & $-1220,85$ \\
\hline Rotated BB6 270 degrees & & -1 & 1,56 & 0 & 0 & 618,61 & $-1233,22$ & $-1220,8$ \\
\hline Rotated BB7 270 degrees & & $-1,77$ & 0 & 0 & 0 & 488,19 & $-972,38$ & $-959,96$ \\
\hline Rotated BB8 270 degrees & & -6 & $-0,6$ & $\mathbf{0}$ & $\mathbf{0}$ & 1014,68 & $-2025,37$ & $-2012,95$ \\
\hline $\begin{array}{c}\text { Rotated Tawn Type } 190 \\
\text { degrees }\end{array}$ & & $-1,52$ & 0,66 & 0 & 0 & 363,97 & $-723,94$ & $-711,51$ \\
\hline $\begin{array}{c}\text { Rotated Tawn Type } 1270 \\
\text { degrees }\end{array}$ & & $-1,77$ & 0,66 & 0 & 0 & 592,44 & $-1180,88$ & $-1168,46$ \\
\hline $\begin{array}{c}\text { Rotated Tawn Type } 290 \\
\text { degrees }\end{array}$ & & $-1,64$ & 0,66 & 0 & 0 & 430,1 & $-856,2$ & $-843,78$ \\
\hline $\begin{array}{c}\text { Rotated Tawn Type } 2270 \\
\text { degrees }\end{array}$ & & $-1,61$ & 0,66 & 0 & 0 & 509,07 & $-1014,13$ & $-1001,71$ \\
\hline Gaussian & & $-0,58$ & & 0 & 0 & 757,22 & $-1512,44$ & $-1506,23$ \\
\hline
\end{tabular}

\section{REFERENCES}

[1] Lardic, S., \& Mignon, V. The impact of oil prices on GDP in European countries: An empirical investigation based on asymmetric cointegration. Energy Policy, 34, 3910-3915 2006. https://doi.org/10.1016/j.enpol.2005.09.019

[2] Pierce, J. L., Enzler, J. J., Fand, D. I., \& Gordon, R. J. The Effects of External Inflationary Shocks. Source: Brookings Papers on Economic Activity, 1974(1), 13-61 1974.. Retrieved from http://www.jstor.org/stable/2534072 
[3] Brown, S. P. A., \& Yücel, M. K. Energy prices and aggregate economic activity: an interpretative survey. The Quarterly Review of Economics and Finance, 42(2), 193-208 2002. https://doi.org/10.1016/S1062-9769(02)00138-2

[4] Basher, S. A., \& Sadorsky, P. Oil price risk and emerging stock markets. Global Finance Journal, 17(2), 224-251 2006. https://doi.org/10.1016/J.GFJ.2006.04.001

[5] Hamilton, J. D. Oil and the Macroeconomy since World War II. Journal of Political Economy, 91(2), $228-248$ 1983. https://doi.org/10.1086/261140

[6] Cong, R.-G., Wei, Y.-M., Jiao, J.-L., \& Fan, Y. Relationships between oil price shocks and stock market: An empirical analysis from China. Energy Policy, 36(9), 3544-3553 2008. https://doi.org/10.1016/J.ENPOL.2008.06.006

[7] Henriques, I., \& Sadorsky, P. Oil prices and the stock prices of alternative energy companies. Energy Economics, 30(3), 998-1010 2008. https://doi.org/10.1016/J.ENECO.2007.11.001

[8] Huang, R. D., Masulis, R. W., \& Stoll, H. R. Energy shocks and financial markets. Journal of Futures Markets, 16(1), 1-27 1996. https://doi.org/10.1002/(SICI)1096-9934(199602)16:1<1::AID-FUT1>3.0.CO;2-Q

[9] Miller, J. I., \& Ratti, R. A. Crude oil and stock markets: Stability, instability, and bubbles. Energy Economics, 31(4), 559-568 2009. https://doi.org/10.1016/J.ENECO.2009.01.009

[10] Papapetrou, E. Oil price shocks, stock market, economic activity and employment in Greece. Energy Economics, 23(5), 511-532, 2001. https://doi.org/10.1016/S0140-9883(01)00078-0

[11]Park, J., \& Ratti, R. A. Oil price shocks and stock markets in the U.S. and 13 European countries. Energy Economics, 30(5), 2587-2608, 2008. https://doi.org/10.1016/J.ENECO.2008.04.003

[12] Sadorsky, P. Oil price shocks and stock market activity. Energy Economics, 21(5), 449-469, 1999. https://doi.org/10.1016/S0140-9883(99)00020-1

[13]Da Silva Filho, O. C., Ziegelmann, F. A., \& Dueker, M. J. Modeling dependence dynamics through copulas with regime switching. Insurance: Mathematics and Economics, 50, 346-356, 2012.

[14] Sukcharoen, K., Zohrabyan, T., Leatham, D., \& Wu, X. Interdependence of oil prices and stock market indices: A copula approach. Energy Economics, 44, 331-339, 2014. https://doi.org/10.1016/J.ENECO.2014.04.012

[15] Shi, W., Li, K. X., Yang, Z., \& Wang, G. Time-varying copula models in theshipping derivatives market. Empirical Economics, 53, 1039-1058, 2017.

[16]Zhu, H.-M., Li, R., \& Li, S.. Modelling dynamic dependence between crude oil prices and Asia-Pacific stock market returns. International Review of Economics \& Finance, 29, 208-223, 2014 https://doi.org/10.1016/J.IREF.2013.05.015

[17] Sklar, M. Fonctions de repartition an dimensions et leurs marges. Publ. inst. statist. univ. Paris, 8, 229-231, 1959. 